\title{
FINER DIOPHANTINE AND REGULARITY PROPERTIES OF 1-DIMENSIONAL PARABOLIC IFS
}

\author{
MARIUSZ URBAŃSKI
}

\begin{abstract}
Recall that a Borel probability measure $\mu$ on $\mathbb{R}$ is called extremal if $\mu$-almost every number in $\mathbb{R}$ is not very well approximable. In this paper, we prove extremality (and implying it the exponentially fast decay property (efd)) of conformal measures induced by 1-dimensional finite parabolic iterated function systems. We also investigate the doubling property of these measures and we estimate from below the Hausdorff dimension of the limit sets of such iterated systems.
\end{abstract}

\section{INTRODUCTION}

This paper is about not very well approximable and badly approximable numbers occuring in the limit set of a 1-dimensional finite parabolic iterated function system. A point $x \in \mathbb{R}$ is called very well approximable if there exist $\delta>0$ and infinitely many integers $p, q \in \mathbb{Z}$, $q \geq 1$, such that

$$
|q x-p| \leq q^{-(1+\delta)}
$$

It is a classical result that the set of all very well approximable numbers has the Lebesgue measure zero but the Hausdorff dimension equal to 1 . Thus the natural question arises about other measures. To be more precise, a Borel measure $\mu$ on $\mathbb{R}$ is called extremal if $\mu$-almost every number in $\mathbb{R}$ is not very well approximable. Barak Weiss has provided in [10] a nice sufficient condition, which we call exponentially fast decay (efd) (see the beginning of Section 3 for its definition) for a Borel probability measure on $\mathbb{R}$ to be extremal. We will use this result frequently. We have originated in [8] to study the extremality property of conformal measures of 1-dimensional regular iterated function systems with special attention paid to the systems generated by the ordinary continued fraction algorithm. For the corresponding results about multi-dimensional systems see [2], [1] and [9] for example. In this paper we continue the topic of 1-dimensional systems focusing this time on finite parabolic iterated function systems. Our ultimate result here, Theorem 5.1, is that the conformal measure of every 1-dimensional finite parabolic iterated function system satisfies the (efd) property, and is consequently extremal.

The second leading theme of this paper concerns badly approximable numbers. Recall that a number $x \in \mathbb{R}$ is badly approximable if there is $C>0$ such that for all $p \in \mathbb{Z}$ and $q \in \mathbb{N}$, we have that

$$
\left|x-\frac{p}{q}\right| \geq \frac{c}{q^{2}}
$$

D. Kleinbock and Barak Weiss provided in [1] a very useful tool to estimate from below the Hausdorff dimension of the set of badly approximable points lying in the topological support 
of a Borel probability measure. Let us describe this tool in our special 1-dimensional context. Following [2] we say that a Borel probability measure $\mu$ on $\mathbb{R}$ is absolutely decaying if there exist a constant $C>0$ and $\alpha>0$ such that for all $x, y \in \mathbb{R}$ and all $r, \varepsilon>0$ it holds

$$
\mu(B(x, r) \cap B(y, \varepsilon r)) \leq C \varepsilon^{\alpha} \mu(B(x, r)) .
$$

The measure $\mu$ is said to satisfy the doubling (Federer) property provided that there is a constant $F>0$ such that

$$
\mu(B(x, 2 r)) \leq F \mu(B(x, r))
$$

for every $x \in \mathbb{R}$ and every $r>0$. Finally, given $s>0$ the measure $\mu$ is said to be $s$-upper geometric if

$$
\mu(B(x, r)) \leq G r^{s}
$$

for some constant $G>0$, all $x \in \mathbb{R}$ and all $r>0$. The result (in our context) of of Kleinbock and Weiss from [1] is given by the following.

Theorem 1.1. If a Borel probability measure $\mu$ on $\mathbb{R}$ is absolutely decaying, s-upper geometric and satisfies the doubling property, then the Hausdorff dimension of the set of badly approximable points lying in the topological support of the measure $\mu$ is greater than or equal to $s$.

In Section 6 we introduce the concept of extendable 1-dimensional finite parabolic iterated function systems and we prove in Section 7 that the Hausdorff dimension of the set of badly approximable points lying in the limit set of such a system is greater than or equal to $h+$ $p_{S}(h-1)>0$, where $h \in(0,1]$ is the Hausdorff dimension of the limit set and $p_{S}>0$ is the parameter describing the local behavior of our system around parabolic points. The idea of the proof is to verify the assumptions of Theorem 1.1 for the $h$-conformal measure $m$. And indeed, the absolute decaying property of $m$ is a rather easy consequence of the (efd) property and the doubling property. That the conformal measure $m$ is $h+p_{S}(h-1)(>0)$-upper geometric follows easily from [7]. The issue is the doubling property, which is interesting itself. The doubling property of conformal measures of 1-dimensional systems was studied in [5]. Here, we provide at the beginning of Section 4 a general sufficient condition for the conformal measure of a 1-dimensional iterated function system to satisfy the doubling property, the condition weaker that those from [5]. As we have already indicated we introduce in Section6 the concept of extendable 1-dimensional finite parabolic iterated function systems, which repalaces the annoying super strong open set condition from [5], and we ultimately prove that each extendable 1-dimensional finite parabolic iterated function system satisfies the doubling property. 


\section{Prelimanaries from Conformal IFS}

Our setting is the following. Let $X$ be a compact subset of a Euclidean space $\mathbb{R}^{d}$ with nonempty interior such that the boundary of $X$ has no isolated points. We consider a countable family of conformal maps $\phi_{i}: X \rightarrow X, i \in I$, where $I$ has at least two elements, satisfying the following conditions.

(1) (Open Set Condition) $\phi_{i}(\operatorname{Int}(X)) \cap \phi_{j}(\operatorname{Int}(X))=\emptyset$ for all $i \neq j$.

(2) $\left|\phi_{i}^{\prime}(x)\right|<1$ everywhere except for finitely many pairs $\left(i, x_{i}\right), i \in I$, for which $x_{i}$ is the unique fixed point of $\phi_{i}$ and $\left|\phi_{i}^{\prime}\left(x_{i}\right)\right|=1$. Such pairs and indices $i$ will be called parabolic and the set of parabolic indices will be denoted by $\Omega$. All other indices will be called hyperbolic.

(3) (extension) There exist an open connected neighbourhood $V$ of $X$ and $s<1$ such that $\forall n \geq 1 \forall \omega=\left(\omega_{1}, \ldots, \omega_{n}\right) \in I^{n}$ if $\omega_{n}$ is a hyperbolic index or $\omega_{n-1} \neq \omega_{n}$, then $\phi_{\omega}$ extends conformally to $V$, maps $V$ into itself and $\left\|\phi_{\omega}^{\prime}\right\| \leq s$.

(4) If $i$ is a parabolic index, then $\bigcap_{n \geq 0} \phi_{i^{n}}(X)=\left\{x_{i}\right\}$ (Thus, the diameters of the sets $\phi_{i^{n}}(X)$ converge to 0.$)$

(5) (Cone Condition) There exist $\alpha, l>0$ such that for every $x \in \partial X \subset \mathbb{R}^{d}$, there exists an open cone $\operatorname{Con}\left(x, u_{x}, \alpha, l\right) \subset \operatorname{Int}(X)$ with vertex $x$ and a central angle of Lebesgue measure $\alpha$, where $\operatorname{Con}\left(x, u_{x}, \alpha, l\right)=\left\{y: 0<\left(y-x, u_{x}\right) \leq \cos \alpha\|y-x\| \leq l\right\}$ and $\left\|u_{x}\right\|=1$

(6) $\exists s<1 \forall n \geq 1 \forall \omega \in I^{n}$ if $\omega_{n}$ is a hyperbolic index or $\omega_{n-1} \neq \omega_{n}$, then $\left\|\phi_{\omega}^{\prime}\right\| \leq s$.

(7) (Bounded Distortion Property) $\exists K \geq 1 \forall n \geq 1 \forall \omega=\left(\omega_{1}, \ldots, \omega_{n}\right) \in I^{n} \forall x, y \in V$ if $\omega_{n}$ is a hyperbolic index or $\omega_{n-1} \neq \omega_{n}$, then

$$
\frac{\left|\phi_{\omega}^{\prime}(y)\right|}{\left|\phi_{\omega}^{\prime}(x)\right|} \leq K
$$

(8) There are constants $L \geq 1$ and $\alpha>0$ such that

$$
|| \phi_{i}^{\prime}(y)|-| \phi_{i}^{\prime}(x)|| \leq L \| \phi_{i}^{\prime}||(\|y-x\|)^{\alpha},
$$

for every $i \in I$ and every pair of points $x, y \in V$.

Call a finite word $\omega=\omega_{1} \omega_{2} \ldots \omega_{n-1} \omega_{n}$ hyperbolic if $\omega_{n}$ is a hyperbolic index or $\omega_{n-1} \neq \omega_{n}$. As an esasy consequence of (7) and (8) we get the following strngtshening of (8).

(9) (Strong Bounded Distortion Property) For every $\varepsilon>0$ there exists $\delta>0$ such that for every hyperbolic word $\omega$, every $x \in X$ and every $y \in B(x, \delta)$, we have

$$
\frac{\left|\phi_{\omega}^{\prime}(y)\right|}{\left|\phi_{\omega}^{\prime}(x)\right|} \leq 1+\varepsilon
$$

The system $S=\left\{\phi_{i}: i \in I\right\}$ is called a conformal iterated function system abbreviated as conformal IFS. If $\Omega=\emptyset$, the system $S$ is called hyperbolic and if $\Omega \neq \emptyset$, it is called parabolic. By $I^{*}$ we denote the set of all finite words with alphabet $I$ and by $I^{\infty}$ all infinite sequences with terms in $I$. It follows from (3) that for every hyperbolic word $\omega, \phi_{\omega}(V) \subset V$. For each $\omega \in I^{*} \cup I^{\infty}$, we define the length of $\omega$ by the uniquely determined relation $\omega \in I^{|\omega|}$. If 
$\omega \in I^{*} \cup I^{\infty}$ and $n \leq|\omega|$, then by $\left.\omega\right|_{n}$ we denote the word $\omega_{1} \omega_{2} \ldots \omega_{n}$. In [MU4], we proved that $\lim _{n \rightarrow \infty} \sup _{|\omega|=n}\left\{\operatorname{diam}\left(\phi_{\omega}(X)\right)\right\}=0$. So, the map $\pi: I^{\infty} \rightarrow X, \pi(\omega)=\bigcap_{n \geq 0} \phi_{\left.\omega\right|_{n}}(X)$, is uniformly continuous. Its range

$$
J=J_{S}=\pi\left(I^{\infty}\right),
$$

the main object of our interest in this paper, is called the limit set of the system $S$. For every integer $q \geq 1$, we denote

$$
S^{q}=\left\{\phi_{\omega}: \omega \in I^{q}\right\} .
$$

Of course, $J_{S^{q}}=J_{S}$ and sometimes in the sequel it will be more convenient to consider an appropriate family of iterates $S^{q}$ of $S$ rather than $S$ itself. If $d \geq 3$, we put $p_{i}=1$ for all $i \in \Omega$. If $d=2$ and $i \in \Omega$, then there are $p_{i} \in\{1,2,3, \ldots\}, a \in \mathbb{C} \backslash\{0\}$ and $a_{n} \in \mathbb{C}$ for every $n \geq p_{i}+2$ such that either

$\phi_{i}(z)=z+a\left(z-x_{i}\right)^{p_{i}+1}+\sum_{n=p_{i}+2}^{\infty} a_{n}\left(z-x_{i}\right)^{n}$ or $\phi_{i}(z)=\bar{z}+a\left(\overline{z-x_{i}}\right)^{p_{i}+1}+\sum_{n=p_{i}+2}^{\infty} a_{n}\left(\overline{z-x_{i}}\right)^{n}$

on a sufficiently small neighbourhood of $x_{i} \in \mathbb{C}$. If $d=1$, we assume that for every $i \in \Omega$ there exist $p_{i} \in(0,+\infty)$ and $a>0$ such that

$$
\phi_{i}(x)=x-\operatorname{asgn}\left(x-x_{i}\right)\left|x-x_{i}\right|^{p_{i}+1}+o\left(\left|x-x_{i}\right|^{p_{i}+1}\right) .
$$

Let

$$
h=\operatorname{HD}\left(J_{S}\right)
$$

be the Hausdorff dimension of the limit set $J_{S}$. A Borel probability measure $m$ on $J$ is called $h$-conformal if and only if

$$
m\left(\phi_{i}(A)\right)=\int_{A}\left|\phi_{i}^{\prime}\right|^{h} d m
$$

for every Borel set $A \subset X$. If an $h$-conformal measure $m$ exists, the system $S$ is called regular and the measure $m$ is unique. The following result has been proved in [7] in the case when $d=1$ and in [4] in the case when $d \geq 2$.

Theorem 2.1. If $S$ is a finite parabolic IFS, then the system $S$ is regular and, consequently, an $h$-conformal measure for $S$ exists. In addition

$$
h>\max \left\{\frac{p_{i}}{p_{i}+1}: i \in \Omega\right\} .
$$

Given two sets $A, B \subset \mathbb{R}^{d}$ let

$$
\operatorname{dist}(A, B)=\inf \{\|y-x\|: x \in A, y \in B\}
$$

and

$$
\operatorname{Dist}(A, B)=\sup \{\|y-x\|: x \in A, y \in B\}
$$

The formulas below have been proved in [4] assuming that $d \geq 2$. If $d=1$, these can be easily derived from formulas established in [7]. Here they are. There exist a constant $Q \geq 1$ and an 
FINER DIOPHANTINE AND REGULARITY PROPERTIES OF 1-DIMENSIONAL PARABOLIC IFS 5

integer $q \geq 0$ such that for every parabolic index $i \in I$, every $j \in I \backslash\{i\}$, and all $n, k \geq 1$ we have that

$$
\begin{gathered}
Q^{-1} n^{-\frac{p_{i}+1}{p_{i}}} \leq \inf _{X}\left\{\left\|\phi_{i^{n} j}^{\prime}(x)\right\|\right\},\left\|\phi_{i^{n} j}^{\prime}\right\|, \operatorname{diam}\left(\phi_{i^{n} j}(X)\right) \leq Q n^{-\frac{p_{i}+1}{p_{i}}}, \\
Q^{-1} n^{-\frac{1}{p_{i}}} \leq \operatorname{dist}\left(x_{i}, \phi_{i^{n} j}(X)\right) \leq \operatorname{Dist}\left(x_{i}, \phi_{i^{n} j}(X)\right) \leq Q n^{-\frac{1}{p_{i}}}, \\
\operatorname{Dist}\left(\phi_{i^{n} j}(X), \phi_{i^{k} j}(X)\right) \leq Q\left|\min \{k, n\}^{-\frac{1}{p_{i}}}-(\max \{k, n\}+1)^{-\frac{1}{p_{i}}}\right|
\end{gathered}
$$

and, furthermore, if $|n-k| \geq q$, then

$$
\operatorname{dist}\left(\phi_{i^{n} j}(X), \phi_{i^{k} j}(X)\right) \geq Q\left|n^{-\frac{1}{p_{i}}}-k^{-\frac{1}{p_{i}}}\right| \text {. }
$$

These formulas along with Theorem 2.1 are sufficient for all the considerations in the last section of [4] to go through regardless whether $d=1$ or $d \geq 2$. A convenient tool to study limit sets of parabolic iterated function systems and corresponding conformal measures is the hyperbolic (see Theorem 5.2 in [3]) system $S^{*}$ associated with $S$. The system $S^{*}$ is given by

$$
S^{*}=\left\{\phi_{i^{n} j}: n \geq 1, i \in \Omega, i \neq j\right\} \cup\left\{\phi_{k}: k \in I \backslash \Omega\right\} .
$$

Thus, $I_{*}$, the countable set of indices or letters for the system $S^{*}$ is

$$
I_{*}=\left\{i^{n} j: n \geq 1, i \in \Omega, i \neq j\right\} \cup\{k: k \in I \backslash \Omega\} .
$$

This system was described and analyzed in [3]. The limit sets generated by the system $S^{*}$ and $S$ differ only by a countable set. If a parabolic system $S$ is regular, in particular if it is finite, then the $h$-conformal measure $m$ for $S$ is obviously $h$-conformal for $S^{*}$.

\section{EXtremality AND (EFD) PROPERTy FOR REgUlar 1-DIMENSIONAL SYSTEMS}

We have proved in [8] the following proposition.

Proposition 3.1. Let $(X, \rho)$ be a metric space and let $\mu$ be a Borel probability measure on $X$. Then the following two conditions are equivalent.

(a)

$$
\begin{gathered}
\exists(\alpha \in(0,1)) \exists(\beta>1) \exists(\xi>0) \forall(x \in X) \forall(r \leq \xi) \\
\mu(B(x, r)) \leq \alpha \mu(B(x, \beta r)) .
\end{gathered}
$$

(b) There exists a Borel set $Y \subset X$ with $\mu(Y)=1$ such that the condition (a) is satisfied with $X$ replaced by $Y$.

Any Borel probability measure satisfying condition (a) or, equivalently, condition (b) of Proposition 3.1, was said in [8] to satisfy the exponentially fast decay (efd) property. The significance of this property, though interesting itself, results from the following fact, essentially proven in [10] and crucial for our approach. 
Theorem 3.2. (B. Weiss) Every Borel probability measure on $\mathbb{R}$ satisfying the (efd) property is extremal.

For every $x \in \mathbb{R}$ and $r>0$ put

$$
B_{+}(x, r)=B(x, r) \cap[x,+\infty) \text { and } B_{-}(x, r)=B(x, r) \cap(-\infty, x] .
$$

We prove in this section the following general condition for the conformal measure of a 1dimensional regular system to satisfy the (efd) property.

Theorem 3.3. Suppose that the 1-dimensional system $S=\left\{\phi_{i}\right\}_{i \in I}$ is regular and denote by $m$ the corresponding h-conformal measure. Assume that there exist four real constants: $\gamma \geq 1$, $\beta>1, \alpha \in(0,1), \kappa \in(0, \min \{\operatorname{dist}(X, \partial V),|X|\})$, and a finite set $F \subset I$ such that

$$
m(B(x, r)) \leq \alpha m(B(x, \beta r))
$$

for all $i \in I \backslash F$, all $x \in \phi_{i}(J)$ and all $r \in\left[\gamma\left\|\phi_{i}^{\prime}\right\|, \kappa\right]$. Then the $h$-conformal measure $m$ satisfies the (efd) property and is consequently extremal. that

Proof. Fix an integer $s \geq 0$ so large that $3 K^{h} \alpha^{s}<1$. A straightforward induction gives

$$
m(B(x, r)) \leq \alpha^{s} m\left(B\left(x, \beta^{s} t\right)\right)
$$

for all $i \in I \backslash F$, all $x \in \phi_{i}(J)$ and all $r \in\left[\gamma\left\|\phi_{i}^{\prime}\right\|, \beta^{-s} \kappa\right]$. Since $\operatorname{supp}(m)=\bar{J}$, we have that $Q:=\inf \{m(B(x, \kappa)): x \in J\}>0$ and, since the measure $m$ has no atoms, there exists $\theta \in\left(0, K^{-1} \min \{1, \kappa,|X|\}\right)$ so small that

$$
m(B(x, K \theta)) \leq \alpha^{s} Q
$$

for all $x \in J$. Fix now $x \in J$ and $r \in(0, \theta)$. Write $x=\pi(\omega)$, where $\omega \in I^{\infty}$, and let $n \geq 0$ be the least integer such that $\left\|\phi_{\omega \mid n}^{\prime}\right\| \leq \theta^{-1} r$. Then $n \geq 1$ and

$$
\left\|\phi_{\left.\omega\right|_{n-1}}^{\prime}\right\|>\theta^{-1} r
$$

Consequently

$$
\gamma \theta^{-1} K r\left\|\phi_{\omega_{n-1}}^{\prime}\right\|^{-1} \geq \gamma \theta^{-1} r\left\|\phi_{\omega_{n}}^{\prime}\right\|^{-1}\left\|\phi_{\omega_{n}}^{\prime}\right\| \geq \gamma\left\|\phi_{\omega_{n}}^{\prime}\right\|
$$

and

$$
K r\left\|\phi_{\left.\omega\right|_{n-1}}^{\prime}\right\|^{-1}<K \theta<\kappa \leq \operatorname{dist}(X, \partial V) .
$$

In view of (3.4) and the choice of $\theta$, we get that

$$
\left|\phi_{\left.\omega\right|_{n-1}}(X)\right| \geq K^{-1}|| \phi_{\left.\omega\right|_{n-1}}^{\prime}|||X| \geq K^{-1} \theta^{-1}|X| r>r .
$$


Hence, the set $\phi_{\left.\omega\right|_{n-1}}(X)$ contains at least one side of the ball $B(x, r)$. So, we may assume without loss of generality that $\phi_{\left.\omega\right|_{n-1}}(X) \supset B_{+}(x, r)$. Combining this with (3.6), we see that

$$
\begin{aligned}
\phi_{\left.\omega\right|_{n-1}}\left(X \cap B\left(\pi\left(\sigma^{n-1}(\omega), K r\left\|\phi_{\left.\omega\right|_{n-1}}^{\prime}\right\|^{-1}\right)\right)\right. & =\phi_{\left.\omega\right|_{n-1}}(X) \cap \phi_{\left.\omega\right|_{n-1}}\left(B\left(\pi\left(\sigma^{n-1}(\omega), K r\left\|\phi_{\left.\omega\right|_{n-1}}^{\prime}\right\|^{-1}\right)\right)\right. \\
& \supset B_{+}(x, r) .
\end{aligned}
$$

Hence, assuming that $\gamma \theta^{-1} \beta^{s} K r\left\|\phi_{\left.\omega\right|_{n-1}}^{\prime}\right\|^{-1}<\kappa$, using (3.5) along with the fact that $\pi\left(\sigma^{n-1}(\omega) \in\right.$ $\phi_{\omega_{n}}(J)$ and also (3.2), we get that

$$
\begin{aligned}
m\left(B_{+}(x, r)\right) & \leq m\left(\phi_{\left.\omega\right|_{n-1}}\left(X \cap B\left(\pi\left(\sigma^{n-1}(\omega)\right), K r\left\|\phi_{\left.\omega\right|_{n-1}}^{\prime}\right\|^{-1}\right)\right)\right) \\
& =\int_{X \cap B\left(\pi\left(\sigma^{n-1}(\omega)\right), K r\left\|\phi_{\left.\omega\right|_{n-1}}^{\prime}\right\|^{-1}\right)}\left|\phi_{\left.\omega\right|_{n-1}}^{\prime}\right|^{h} d m \\
& \leq\left\|\phi_{\left.\omega\right|_{n-1}}^{\prime}\right\|^{h} m\left(B\left(\pi\left(\sigma^{n-1}(\omega)\right), K r\left\|\phi_{\left.\omega\right|_{n-1}}^{\prime}\right\|^{-1}\right)\right) \\
& \leq\left\|\phi_{\left.\omega\right|_{n-1}}^{\prime}\right\|^{h} m\left(B\left(\pi\left(\sigma^{n-1}(\omega)\right), \gamma \theta^{-1} K r\left\|\phi_{\left.\omega\right|_{n-1}}^{\prime}\right\|^{-1}\right)\right) \\
& \leq \alpha^{s}\left\|\phi_{\left.\omega\right|_{n-1}}^{\prime}\right\|^{h} m\left(B\left(\pi\left(\sigma^{n-1}(\omega)\right), \beta^{s} \gamma \theta^{-1} K r\left\|\phi_{\left.\omega\right|_{n-1}}^{\prime}\right\|^{-1}\right)\right) \\
& \leq K^{h} \alpha^{s} m\left(\phi_{\left.\omega\right|_{n-1}}\left(B\left(\pi\left(\sigma^{n-1}(\omega)\right), \beta^{s} \gamma \theta^{-1} K r\left\|\phi_{\left.\omega\right|_{n-1}}^{\prime}\right\|^{-1}\right)\right)\right) \\
& \leq K^{h} \alpha^{s} m\left(B\left(x, \beta^{s} \gamma \theta^{-1} K r\right)\right) .
\end{aligned}
$$

If, on the other hand, $\gamma \theta^{-1} \beta^{s} K r\left\|\phi_{\left.\omega\right|_{n-1}}^{\prime}\right\|^{-1} \geq \kappa$, then using (3.3), (3.6) and (3.7), we obtain

$$
\begin{aligned}
m\left(B\left(x, \beta^{s} \gamma \theta^{-1} K r\right)\right) & \geq m\left(\phi_{\left.\omega\right|_{n-1}}\left(B\left(\pi\left(\sigma^{n-1}(\omega)\right), \kappa\right)\right)\right) \\
& \geq K^{-h}\left\|\phi_{\left.\omega\right|_{n-1}}^{\prime}\right\|^{h} m\left(B\left(\pi\left(\sigma^{n-1}(\omega)\right), \kappa\right)\right) \\
& \geq K^{-h} \alpha^{-s}|| \phi_{\left.\omega\right|_{n-1}}^{\prime} \|^{h}\left(B\left(\pi\left(\sigma^{n-1}(\omega)\right), K \theta\right)\right) \\
& \left.\geq K^{-h} \alpha^{-s} m\left(\phi_{\left.\omega\right|_{n-1}}\left(B\left(\pi\left(\sigma^{n-1}(\omega)\right), K \theta\right) \cap X\right)\right)\right) \\
& \geq K^{-h} \alpha^{-s} m\left(\phi_{\left.\omega\right|_{n-1}}\left(B\left(\pi\left(\sigma^{n-1}(\omega)\right), K r\left\|\phi_{\left.\omega\right|_{n-1}}^{\prime}\right\|^{-1}\right) \cap X\right)\right) \\
& \geq K^{-h} \alpha^{-s} m\left(B_{+}(x, r)\right) .
\end{aligned}
$$

In any case, (3.8) always holds. Now let $y \in \bar{J}$ be the infimum of all $z \in B_{-}(x, r)$ for which (3.8) holds with $x$ replaced by $z$. Since the measure $m$ has no atoms, (3.8) also holds for $y$. If $J \cap(x-r, y)=\emptyset$, then $B(x, r) \cap J=J \cap\left(B_{+}(y, r) \cup B_{+}(x, r)\right)$, and consequently

$$
\begin{aligned}
m(B(x, r) & \leq m\left(B_{+}(y, r)\right)+m\left(B_{+}(x, r)\right) \\
& \leq K^{h} \alpha^{s}\left(m\left(B\left(y, \beta^{s} \gamma \theta^{-1} K r\right)\right)+m\left(B\left(x, \beta^{s} \gamma \theta^{-1} K r\right)\right)\right) \\
& \leq 2 K^{h} \alpha^{s} m\left(B\left(x,\left(1+\beta^{s} \gamma \theta^{-1} K\right) r\right)\right) .
\end{aligned}
$$

If $J \cap(x-r, y) \neq \emptyset$, then set $w=\sup (J \cap(x-r, y))$. There then exists an infinite sequence $\left\{w_{k}\right\}_{k=1}^{\infty}$ of points (not necessarily distinct) in $J \cap(x-r, y)$ converging to $w$ such that for 
every $k \geq 1$ the formula (3.8) is satisfied with $B_{+}(x, r)$ replaced by $B_{-}\left(w_{k}, r\right)$. Since $m$ is atomless, (3.8) therefore holds also for $B_{+}(x, r)$ replaced by $B_{-}(w, r)$. Since $J \cap B(x, r) \subset$ $\left.B_{(} w, r\right) \cup B_{+}(y, r) \cup B_{+}(x, r)$, we thus get

$$
\begin{aligned}
m(B(x, r)) & \leq m\left(B_{-}(w, r) \cup B_{+}(y, r) \cup B_{+}(x, r)\right) \\
& \leq K^{h} \alpha^{s}\left(m\left(B\left(w, \beta^{s} \gamma \theta^{-1} K r\right)\right)+m\left(B\left(y, \beta^{s} \gamma \theta^{-1} K r\right)\right)\right)+m\left(B\left(x, \beta^{s} \gamma \theta^{-1} K r\right)\right) \\
& \leq 3 K^{h} \alpha^{s} m\left(B\left(x,\left(1+\beta^{s} \gamma \theta^{-1} K\right) r\right)\right) .
\end{aligned}
$$

We are done.

\section{The Doubling Property for Regular 1-Dimensional Systems}

A Borel probability measure $\nu$ in a metric space $Y$ is said to satisfy the doubling (Federer) property provided that there exists a constant $C \geq 1$ such that for every $x \in Y$ and every radius $r>0$ we have that $\nu(B(x, 2 r)) \leq C \nu(B(x, r))$. In [5] (see Theorem 2.1) we have provided a sufficient condition for the $h$-conformal measure of a regular iterated function system to satisfy the doubling property. In here, in the setting of 1-dimensional systems, we want to get rid of this rather restrictive and annoying condition replacing it by condition (a) in the next proposition. In order to formulate it we need one definition. Given a conformal iterated function system $S=\left\{\phi_{i}: X \rightarrow X\right\}_{i \in I}, X \subset V$, a pair $(Y, W)$ is called $S$-eligible provided that the following conditions are satisfied.

(a) $Y \subset X$ is a compact connected set and $W \subset V$ is an open connected set.

(b) $Y \subset W$.

(c) $\phi_{i}(Y) \subset Y$ and $\phi_{i}(W) \subset W$ for all $i \in i$.

Obviously $S^{\prime}=\left\{\phi_{i}: Y \rightarrow Y\right\}_{i \in I}, Y \subset W$, is a conformal iterated function system, $J_{S^{\prime}}=J_{S}$, and conformal measures for $S$ and $S^{\prime}$ (if at least one exists) coincide. $S^{\prime}$ is called an eligible version of $S$. Let $a$ and $b$ be respectively the left hand endpoint of $Y$ and the right hand endpoint of $Y$. We shall prove the following.

Proposition 4.1. Suppose that $S=\left\{\phi_{i}\right\}_{i \in I}$ is a regular 1-dimensional iterated function system and denote by $m$ the corresponding h-conformal measure. Suppose also that there exist an $S$-eligible pair $(X, V)$ and constants $\gamma \geq 1, \rho \in(0, \operatorname{dist}(X, \partial V)$ and $C>0$ with the following properties:

(a) $\forall\left(\omega \in I^{*}\right) \forall(r \in(0, \rho)$ $m\left(\phi_{\omega}\left(B_{+}(b, r)\right)\right) \leq C m\left(\phi_{\omega}\left(B_{-}(b, r)\right)\right) \quad$ and $m\left(\phi_{\omega}\left(B_{-}(a, r)\right)\right) \leq C m\left(\phi_{\omega}\left(B_{+}(b, r)\right)\right)$.

(b) $\forall(i \in I) \forall\left(x \in \phi_{i}(J)\right) m\left(B\left(x, 2 \gamma \operatorname{diam}\left(\phi_{i}(X)\right)\right) \leq C\left\|\phi_{i}^{\prime}\right\|^{h}\right.$.

(c) $\left.\forall(i \in I) \forall\left(x \in \phi_{i}(J)\right) \forall\left(r \geq \operatorname{diam}\left(\phi_{i}(X)\right)\right)\right) m(B(x, 2 r)) \leq C m(B(x, r))$.

Then the measure $m$ satisfies the doubling property. 
Proof. It follows from the Strong Bounded Distortion Property that there exists $R \in(0, \rho)$ such that for all $\omega \in I^{*}$, all $x \in X$, and all $y \in B(x, 2 R)$, we have that

$$
\frac{3}{4} \leq \frac{\left|\phi_{\omega}^{\prime}(y)\right|}{\left|\phi_{\omega}^{\prime}(x)\right|} \leq \frac{4}{3}
$$

Fix now an arbitrary point $x \in J$ and radius $r \in(0, R / 2)$. Write $x=\pi(\omega), \omega \in I^{\infty}$. There then exists the least $n \geq 0$ such that $2 r\left\|\phi_{\left.\omega\right|_{n}}^{\prime}\right\|^{-1}<R$. Then $B\left(\pi\left(\sigma^{n}(\omega)\right), 2 r\left\|\phi_{\left.\omega\right|_{n}}^{\prime}\right\|^{-1}\right) \subset V$, and it follows from (4.1) that

$$
\phi_{\left.\omega\right|_{n}}\left(B\left(\pi\left(\sigma^{n}(\omega)\right), \frac{3}{4} r\left\|\phi_{\left.\omega\right|_{n}}^{\prime}\right\|^{-1}\right)\right) \subset B(x, r) .
$$

Hence,

$$
m(B(x, r)) \geq\left(\frac{3}{4}\right)^{h}\left\|\phi_{\left.\omega\right|_{n}}^{\prime}\right\|^{h} m\left(B\left(\pi\left(\sigma^{n}(\omega)\right), \frac{3}{4} r\left\|\phi_{\left.\omega\right|_{n}}^{\prime}\right\|^{-1}\right)\right)
$$

Also

$$
\phi_{\left.\omega\right|_{n}}\left(B\left(\pi\left(\sigma^{n}(\omega)\right), \frac{3}{2} r\left\|\phi_{\left.\omega\right|_{n}}^{\prime}\right\|^{-1}\right)\right) \subset B\left(x, \frac{9}{8} r\right) .
$$

Since $2 r\left\|\phi_{\left.\omega\right|_{n}}^{\prime}\right\|^{-1}<R$, it follows from condition (a) that

$$
m\left(\phi_{\left.\omega\right|_{n}}\left(B\left(\pi\left(\sigma^{n}(\omega)\right), \frac{3}{2} r\left\|\phi_{\left.\omega\right|_{n}}^{\prime}\right\|^{-1}\right)\right)\right) \leq(1+C) m\left(\phi_{\left.\omega\right|_{n}}\left(X \cap B\left(\pi\left(\sigma^{n}(\omega)\right), \frac{3}{2} r\left\|\phi_{\omega_{n}}^{\prime}\right\|^{-1}\right)\right)\right) .
$$

Therefore, applying (4.3), we get that

$$
\begin{aligned}
m\left(B\left(x, \frac{9}{8} r\right)\right) & \leq(1+C)\left(\frac{4}{3}\right)^{h}\left\|\phi_{\left.\omega\right|_{n}}^{\prime}\right\|^{h} m\left(X \cap B\left(\pi\left(\sigma^{n}(\omega)\right), \frac{3}{2} r\left\|\phi_{\omega_{n}}^{\prime}\right\|^{-1}\right)\right) \\
& =(1+C)\left(\frac{4}{3}\right)^{h}\left\|\phi_{\left.\omega\right|_{n}}^{\prime}\right\|^{h} m\left(X \cap B\left(\pi\left(\sigma^{n}(\omega)\right), \frac{3}{2} r\left\|\phi_{\left.\omega_{n}\right|_{n}}^{\prime}\right\|^{-1}\right)\right) .
\end{aligned}
$$

It follows from minimality of $n \geq 0$ that $2 r\left\|\phi_{\left.\omega\right|_{n+1}}^{\prime}\right\|^{-1} \geq R$. So,

$$
\frac{3}{4} r\left\|\phi_{\left.\omega\right|_{n}}^{\prime}\right\|^{-1} \geq \frac{3}{8} R\left\|\phi_{\left.\omega\right|_{n}}^{\prime}\right\|^{-1}\left\|\phi_{\left.\omega\right|_{n+1}}^{\prime}\right\| \geq \frac{3}{8} K^{-1} R\left\|\phi_{\omega_{n+1}}^{\prime}\right\|
$$

Suppose first that

$$
\frac{3}{4} r\left\|\phi_{\omega \mid n}^{\prime}\right\|^{-1} \geq \gamma \operatorname{diam}\left(\phi_{\omega_{n+1}}(X)\right)
$$

Since $\pi\left(\sigma^{n}(\omega)\right)=\phi_{\omega_{n+1}}\left(\pi\left(\sigma^{n+1}(\omega)\right)\right)$, it therefore follows from item (c) that

$$
m\left(B\left(\pi\left(\sigma^{n}(\omega)\right), \frac{3}{2} r\left\|\phi_{\left.\omega\right|_{n}}^{\prime}\right\|^{-1}\right)\right) \leq C m\left(B\left(\pi\left(\sigma^{n}(\omega)\right), \frac{3}{4} r\left\|\phi_{\left.\omega\right|_{n}}^{\prime}\right\|^{-1}\right)\right) .
$$

Hence, combining this along with (4.4) and (4.2), we obtain

$$
\left.m\left(B\left(x, \frac{9}{8} r\right)\right) \leq C(1+C)\right)\left(\frac{4}{3}\right)^{2 h} m(B(x, r)) .
$$


Looking at (4.5) we may now assume that

$$
\frac{3}{8} K^{-1} R\left\|\phi_{\omega_{n+1}}^{\prime}\right\| \leq \frac{3}{4} r\left\|\phi_{\left.\omega\right|_{n}}^{\prime}\right\|^{-1} \leq \gamma \operatorname{diam}\left(\phi_{\omega_{n+1}}(X)\right)
$$

Using the right-hand side of this formula and applying (4.4) along with item (b), we obtain

$$
\begin{aligned}
m\left(B\left(x, \frac{9}{8} r\right)\right) & \leq(1+C)\left(\frac{4}{3}\right)^{h} m\left(B\left(\pi\left(\sigma^{n}(\omega)\right), 2 \gamma \operatorname{diam}\left(\phi_{\omega_{n+1}}(X)\right)\right)\right) \\
& \leq C(1+C)\left(\frac{4}{3}\right)^{h}\left\|\phi_{\omega_{n}}^{\prime}\right\|^{h}\left\|\phi_{\omega_{n+1}}^{\prime}\right\|^{h} .
\end{aligned}
$$

Since $\operatorname{supp}(m)=\bar{J}$, the number

$$
M=\inf \left\{m\left(B\left(\left(z, \frac{3 R}{8 K}\right)\right): z \in \bar{J}\right\}\right.
$$

is finite. Now using the left-hand side of (4.7) and (4.2), we get that

$$
\begin{aligned}
m(B(x, r)) & \geq\left(\frac{3}{4}\right)^{h}\left\|\phi_{\left.\omega\right|_{n}}^{\prime}\right\|^{h} m\left(B\left(\pi\left(\sigma^{n}(\omega)\right), \frac{3}{8} K^{-1} R\left\|\phi_{\omega_{n+1}}^{\prime}\right\|\right)\right) \\
& \geq\left(\frac{3}{4}\right)^{h}\left\|\phi_{\left.\omega\right|_{n}}^{\prime}\right\|^{h} m\left(\phi_{\omega_{n+1}}\left(B\left(\pi\left(\sigma^{n+1}(\omega)\right), \frac{3 R}{8 K}\right)\right)\right) \\
& \geq\left(\frac{3}{4 K}\right)^{h}\left\|\phi_{\left.\omega\right|_{n}}^{\prime}\right\|^{h}\left\|\phi_{\omega_{n+1}}^{\prime}\right\|^{h} m\left(B\left(\pi\left(\sigma^{n+1}(\omega)\right), \frac{3 R}{8 K}\right)\right) \\
& \geq M\left(\frac{3}{4 K}\right)^{h}\left\|\phi_{\omega_{n}}^{\prime}\right\|^{h}\left\|\phi_{\omega_{n+1}}^{\prime}\right\|^{h} .
\end{aligned}
$$

Along with (4.8), this gives that

$$
m\left(B\left(x, \frac{9}{8} r\right)\right) \leq C(1+C) M^{-1}\left(\frac{16 K}{9}\right)^{h} m(B(x, r)) .
$$

This inequality and (4.6) complete the proof.

\section{Extremality and the (efd) Property for 1-Dimesional Parabolic Iterated FunCTION SySTEMS}

The result of this section, and one of the two main results of the whole paper, is the following.

Theorem 5.1. The h-conformal measure of a finite 1-dimensional parabolic iterated function system satisfies the (efd) property, and is consequently extremal.

Proof. Let $S=\left\{\phi_{i}: X \rightarrow X\right\}_{i \in I}$ be our parabolic system, and let $m$ be the corresponding conformal measure. The idea of the proof is to check that the assumptions of Theorem 3.3 are satisfied for the associated hyperbolic system $S^{*}$. Since $I$ is finite, the set of hyperbolic elements from $I$ in $I_{*}$ is also finite, and it is therefore sufficient to verify (3.1) for the maps 
of the form $\phi_{j^{k}}$, where $i, j \in I, i \neq j, j$ is a parabolic element and $k \geq 2$ is an integer which will be assumed appropriately large in the course of the proof. Fix $\gamma=2 \max \{1,|X|\}$. Fix then $i$ and $j$ as in the previous sentence, $x \in \phi_{j^{k}}(X)$, and $r \in\left[\gamma\left\|\phi_{j^{k}}^{\prime}\right\|, \kappa\right]$, where $\kappa>0$ will be assume sufficiently small in the course of the proof and $\gamma \geq 1$ like $k$ will be assumed appropriately large in the course of the proof. Let

$$
a=\min \left\{l \geq 0: B(x, r) \cap \phi_{j^{l}}(X) \neq \emptyset\right\}, b=\sup \left\{l \geq 0: B(x, r) \cap \phi_{j^{l} i}(X) \neq \emptyset\right\}
$$

and

$$
c=\min \left\{l \geq 0: B(x, \beta r) \supset \phi_{j^{l} i}(X)\right\}, d=\sup \left\{l \geq 0: B(x, \beta r) \supset \phi_{j^{l}}(X)\right\} .
$$

Assume without loss of generality that the (parabolic) fixed point of the parabolic map $\phi_{j}$ is equal to 0 and that $\phi_{j i}(X) \subset(0,+\infty)$. Put $p=p_{j}$. Observe that if $k \geq 2$ and $\gamma$ are large enough, then

$$
a^{-\frac{1}{p}} \asymp x+r \text { and } c^{-\frac{1}{p}} \asymp x+\beta r .
$$

Iff $x-r>0$ or $x-\beta r>0$, then respectively

$$
b^{-\frac{1}{p}} \asymp x-r \text { and } d^{-\frac{1}{p}} \asymp x-\beta r \text {. }
$$

Now, the Mean Value Theorem produces three points $z \in(x-r, x+r), y \in(x-\beta r, x-r)$ and $w \in(x+r, x+\beta r)$ such that

$$
\begin{aligned}
(x+r)^{(p+1) h-p}-(x-r)^{(p+1) h-p} & \asymp r z^{(p+1)(h-1)}, \\
(x-r)^{(p+1) h-p}-(x-\beta r)^{(p+1) h-p} & \asymp r y^{(p+1)(h-1)}, \\
(x+\beta r)^{(p+1) h-p}-(x+r)^{(p+1) h-p} & \asymp r w^{(p+1)(h-1)} .
\end{aligned}
$$

The choice of integers $a, b, c, d$ along with (5.1) gives us that

$$
\begin{aligned}
m(B(x, r)) & \asymp \sum_{l=a}^{b}\left\|\phi_{j^{l} i}^{\prime}\right\|^{h} \asymp \sum_{l=a}^{b} l^{-\frac{p+1}{p} h} \asymp a^{\frac{p-(p+1) h}{p}}-b^{\frac{p-(p+1) h}{p}} \\
& \asymp(x+r)^{(p+1) h-p}-(x-r)^{(p+1) h-p}
\end{aligned}
$$

and

$$
\begin{aligned}
m(B(x, \beta r) \backslash B(x, r)) & =m((x-\beta r, x-r) \cup(x+r, x+\beta r)) \asymp \sum_{l=c}^{a}\left\|\phi_{j^{l} i}^{\prime}\right\|^{h}+\sum_{l=b}^{d}\left\|\phi_{j^{l} i}^{\prime}\right\|^{h} \\
& \asymp c^{\frac{p-(p+1) h}{p}}-a^{\frac{p-(p+1) h}{p}}+b^{\frac{p-(p+1) h}{p}}-d^{\frac{p-(p+1) h}{p}}
\end{aligned}
$$

Now observe that verifying formula (3.1) is equivalent to verifying the formula

$$
m(B(x, \beta r) \backslash B(x, r)) \succeq m(B(x, r)) .
$$

In order to do it consider two cases.

Case $1^{0}: x-\beta r>0$.

Using then (5.4) and (5.3), we get

$$
m(B(x, r)) \asymp r z^{(p+1)(h-1)} \preceq r(x-r)^{(p+1)(h-1)} .
$$


Using in turn (5.5), (5.3), and (5.2), we obtain

$$
\begin{aligned}
m(B(x, \beta r) \backslash B(x, r)) & \asymp r w^{(p+1)(h-1)}-(x-r)^{(p+1) h-p}+(x-r)^{(p+1) h-p}-(x-\beta r)^{(p+1) h-p} \\
& \succeq r y^{(p+1)(h-1)} \succeq r(x-r)^{(p+1)(h-1)} .
\end{aligned}
$$

Hence, we are done in this case.

Case $2^{0}: x-\beta r \leq 0$ :

Then $x+r \leq(1+\beta) r$ and $x+\beta r \leq 2 \beta r$. Thus, in view of (5.4), we get

$$
m(B(x, r)) \preceq \sum_{s=a}^{\infty} s^{-\frac{p+1}{p} h} \asymp(x+r)^{(p+1) h-p} \preceq r^{(p+1) h-p} .
$$

Now it follows from (5.2) and (5.3) that

$$
\begin{aligned}
m(B(x, \beta r) \backslash B(x, r)) & \geq m([x+r, x+\beta r)) \succeq(x+\beta r)^{(p+1) h-p}-(x+r)^{(p+1) h-p} \\
& \asymp r w^{(p+1)(h-1)} \geq r(x+\beta r)^{(p+1)(h-1)} \\
& \geq r r^{(p+1)(h-1)}=r^{(p+1) h-p} .
\end{aligned}
$$

Hence we are also done in this case, and the proof of our theorem is complete.

\section{The Doubling Property for 1-Dimensional Parabolic IFS}

As a fairly immediate consequence of reasonings contained in [3], which are stated only in the case when $d \geq 2$ but, as was explained in the paragraph following formula (2.4), which go through with essentially no modifications in the case when $d=1$ as well, we shall prove the following.

Lemma 6.1. If $S=\left\{\phi_{i}: X \rightarrow X\right\}_{i \in I}$ is an arbitrary finite parabolic iterated function system, then the condition (b) from Proposition 4.1 is satisfied for the hyperbolic system $S^{*}$, i.e. for every $\gamma \geq 1$ there exists $C \geq 1$ such that for every $\omega \in I_{*}$ and every $x \in \phi_{\omega}(J)$,

$$
m\left(B\left(x, 2 \gamma \operatorname{diam}\left(\phi_{\omega}(X)\right)\right)\right) \leq C\left\|\phi_{\omega}^{\prime}\right\|^{h},
$$

where $m$ is the unique $h$-conformal measure for $S$ and $S^{*}$.

Proof. Since the set $I$ is finite, it suffices to prove (6.1) for all the elements of $\omega \in I_{*}$ of the form $i^{n} j$, where $i$ is a parabolic element, $j \neq i$, and $n \geq 1$ is large enough. Inspecting the proof of Lemma 4.7 from [4], we see that all we need to do is to show that $\Sigma_{2} \preceq n^{-\frac{p_{i}+1}{p_{i}} h}$. And indeed, if $n \geq 1$ is large enough, then $\frac{1}{2} n^{-\frac{1}{p_{i}}} \leq \eta \leq 2 n^{-\frac{1}{p_{i}}}$, where $\eta>0$ is taken from the proof of Lemma 4.7 in [4]. But then, regardless whether $h \geq 1$ or $h \leq 1$, we conclude from the third bottom line of page 251 of [4], that

$$
\Sigma_{2} \preceq n^{-\frac{p_{i}+1}{p_{i}}} n^{-\frac{1}{p_{i}}\left(p_{i}+1\right)(h-1)}=n^{-\frac{p_{i}+1}{p_{i}} h}
$$

and we are done. 
Lemma 6.2. If $S$ is a finite 1-dimensional parabolic iterated function system and $m$ is the corresponding h-conformal measure, then the condition (c) of Proposition 4.1 is satisfied for the hyperbolic system $S^{*}$.

Proof. We shall use the notation and formulas established in the proof of Theorem 5.1 (assuming $\beta=2$ ) before this proof was split into cases. And indeed, since $h \leq 1$, it follows from (5.4) and (5.3) that $m(B(x, r)) \asymp r z^{(p+1)(h-1)} \succeq r(x+r)^{(p+1)(h-1)}$. It also follows from (5.3) that $(x+2 r)^{(p+1) h-p}-(x+r)^{(p+1) h-p} \leq r(x+r)^{(p+1)(h-1)}$. Therefore, we conclude from (5.5) and (5.4) that in order to prove our lemma, it suffices to show that

$$
b^{\frac{p-(p+1) h}{p}}-d^{\frac{p-(p+1) h}{p}} \preceq a^{\frac{p-(p+1) h}{p}}-b^{\frac{p-(p+1) h}{p}}
$$

or equivalently that

$$
b^{\frac{p-(p+1) h}{p}} \preceq a^{\frac{p-(p+1) h}{p}}+d^{\frac{p-(p+1) h}{p}} .
$$

But since $p-(p+1) h<0$, it follows from (5.2) and (5.1) that if $x>r$, then

$$
b^{\frac{p-(p+1) h}{p}} \preceq a^{\frac{p-(p+1) h}{p}}
$$

and we are done in this case. If $x \leq r$, then $b=+\infty$ and (6.2) is trivially true. The proof is complete.

Let $S=\left(\left\{\phi_{i}: X \rightarrow X\right\}_{i \in I}, V\right)$ be a finite 1-dimensional parabolic iterated function system. Denote by $\hat{J}$ the convex hull of $J$, i.e. the least closed segment containing $J$. Obviously $\left(\left\{\phi_{i}: \hat{J} \rightarrow \hat{J}\right\}_{i \in I}, V\right)$ is an eligible version of $S$. Denote the left-hand endpoint of $\hat{J}$ by $a$ and the right-hand endpoint by $b$. Notice that there exists exactly one $i_{a} \in I$ such that $a \in \phi_{i_{a}}(\hat{J})$ and exactly one $i_{b} \in I$ such that $b \in \phi_{i_{a}}(\hat{J})$. Rename the set $I$ so that $i_{a}=a$ and $i_{b}=b$. Passing to $S^{2}$, the second iterate of $S$, we may assume without loss of generality that $\phi_{a}(a)=a$ and $\phi_{b}(b)=b$. We call the parabolic system $S$ extendable if one of the following conditions is satisfied:

(ae) $\hat{J}$ is a proper subset of $X$

(be) $\hat{J}=X$, both elements $a$ and $b$ are hyperbolic and $\left|\phi_{a}^{\prime}(a)\right|=\left|\phi_{b}^{\prime}(b)\right|$.

(ce) $\hat{J}=X$, both elements $a$ and $b$ are parabolic and $p_{a}=p_{b}$.

We shall prove the following.

Lemma 6.3. If a finite 1-dimensional parabolic iterated function system $S=\left(\left\{\phi_{i}: X \rightarrow\right.\right.$ $\left.X\}_{i \in I}, V\right)$ is extendable, then the condition ( a) from Proposition 4.1 is satisfied for the hyperbolic system $S^{*}$.

Proof. If both $a, b \in \operatorname{Int} X$, then $\left(\left\{\phi_{i}: \hat{J} \rightarrow \hat{J}\right\}_{i \in I}, X\right)$ is an eligible version of $S$ and condition (a) from Proposition 4.1 is satisfied since $\phi_{\omega}(\operatorname{Int}(X) \backslash \hat{J}) \cap J=\emptyset$ for all $\omega \in I^{*}$. So, keep the assumption that $\hat{J}$ is a proper subset of $X$ but assume that $\hat{J}$ and $X$ have 
exactly one common endpoint. Without loss of generality we may assume that this is the right-hand endpoint $b$. Again, since $\phi_{\omega}(\operatorname{Int}(X) \backslash \hat{J}) \cap J=\emptyset$ for all $\omega \in I^{*}$, condition (a) from Proposition 4.1 is satisfied by the left-hand endpoint $a$. Now fix an arbitrary finite word $\omega \in I^{*}$ and write $\omega=\tau b^{n}, n \geq 0, \tau_{|\tau|} \neq b$ (allowing $\tau=\emptyset$ ). Put $|\tau|=q$. Consider the segment $\phi_{\tau_{q}}\left(\phi_{b^{n}}\left(B_{+}(b, r)\right)\right)$. If $\tau_{q}=\emptyset$, then $\phi_{b^{n}}\left(B_{+}(b, r)\right) \cap J=\emptyset$, and we are done. So, suppose that $\tau_{q} \in I$. Since $\tau_{q} \neq b, \phi_{\tau_{q}}(b) \in \operatorname{Int}(\hat{J})$. If $\phi_{\tau_{q}}(b)=\phi_{i}(c)$, where $c$ is the left-hand endpoint of $X$ and $i \in I$, then taking $r>0$ universally sufficiently small, the open set condition implies that $\phi_{\tau_{q}}\left(B_{+}(b, r)\right) \subset \phi_{i}(X \backslash \hat{J})=\phi_{i}(\operatorname{Int}(X) \backslash \hat{J}) \cup\left\{\phi_{i}(c)\right\} \subset X$. Since $\phi_{i}(\operatorname{Int}(X) \backslash \hat{J}) \cap J=\emptyset$, we conclude that $\phi_{\omega}\left(B_{+}(b, r)\right) \cap J \subset\left\{\phi_{i}(b)\right\}$. Since the conformal measure $m$ has no atoms, we are done in this case. We similarly arrive in the same conclusion if $\phi_{\tau_{q}}(b)$ is no endpoint of any interval $\phi_{i}(X), i \neq \tau_{q}$. So, suppose that $\phi_{\tau_{q}}(b)=\phi_{j}(b)$ for some $j \in I \backslash\left\{\tau_{q}\right\}$. Since $\phi_{\tau_{q}}(b) \neq b$ and $\phi_{b}(b)=b$, we have that $j \neq b$. Notice also that regardless whether $b$ is a parabolic fixed point (see Lemma 5.1 in [7] for this case) or a repelling fixed point, there exists a constant $C_{1} \geq 1$ and $\kappa>0\left(\kappa=h\right.$ in the repelling case and $\kappa=p_{b}+\left(p_{b}-1\right) h$ in the parabolic case) such that for all $s>0$ small enough, we have that

$$
C_{1}^{-1} s^{\kappa} \leq m\left(B_{-}(b, s)\right) \leq C_{1} s^{\kappa} .
$$

Also observe (use formulas (2.2)-(2.5) in the parabolic case) that regardless of whether $b$ is a parabolic fixed point or a repelling fixed point, there exists a constant $C_{2} \geq 1$ such that

$$
C_{2}^{-1} \leq \frac{\left|\phi_{b}^{n}(b+s)-b\right|}{\left|\phi_{b}^{n}(b-s)-b\right|} \leq C_{2}
$$

for all $n \geq 1$ and all $s>0$ sufficiently small. Now, since $\phi_{j}(\hat{J})$ and $\phi_{\tau_{q}}\left(B_{+}(b, R)\right)$ overlap with the common endpoint $\phi_{\tau_{q}}(b)=\phi_{j}(b)(R>0$ fixed), there exists a unique positive-valued function $H_{b}$ defined on $[0, R]$ (assuming that $R>0$ is small enough) such that $\phi_{\tau_{q}}(b+s)=$ $\phi_{j}\left(b-H_{b}(s)\right)$. Now, for every $s \in[0, R]$, we have

$$
\left|\phi_{\tau_{q}}(b+s)-\phi_{\tau_{q}}(b)\right| \leq 2\left|\phi_{\tau_{q}}^{\prime}(b)\right| s
$$

and

$$
\left|\phi_{\tau_{q}}(b+s)-\phi_{\tau_{q}}(b)\right|=\left|\phi_{j}\left(b-H_{b}(s)\right)-\phi_{j}(b)\right| \geq \frac{1}{2}\left|\phi_{j}^{\prime}(b)\right| H_{b}(s) \mid .
$$

Hence,

$$
H_{b}(s) \leq A s
$$

where $A=4 \max \left\{\left|\phi_{i}^{\prime}(b)\right|: i \in I\right\} \min ^{-1}\left\{\left|\phi_{i}^{\prime}(b)\right|: i \in I\right\}$. Using (6.5) and (6.4), we get that

$$
\begin{aligned}
\phi_{\tau b^{n}}\left(B_{+}(b, r)\right) & =\phi_{\left.\tau\right|_{q-1}} \circ \phi_{\tau_{q}}\left(B_{+}\left(b, \phi_{b}^{n}(b+r)-b\right)\right)=\phi_{\left.\tau\right|_{q-1}} \circ \phi_{j}\left(B_{-}\left(b, H_{b}\left(\phi_{b}^{n}(b+r)-b\right)\right)\right) \\
& \subset \phi_{\left.\tau\right|_{q-1}} \circ \phi_{j}\left(B_{-}\left(b, A\left|\phi_{b}^{n}(b+r)-b\right|\right)\right) \\
& \subset \phi_{\left.\tau\right|_{q-1}} \circ \phi_{j}\left(B_{-}\left(b, A C_{2}\left|\phi_{b}^{n}(b-r)-b\right|\right)\right) .
\end{aligned}
$$


It therefore follows from $h$-conformality of the measure $m$ and (6.3) that

$$
m\left(\phi_{\tau b^{n}}\left(B_{+}(b, r)\right)\right) \leq\left\|\phi_{\tau_{q-1}}^{\prime}\left|\left\|^{h}\right\| \phi_{j}^{\prime} \|^{h} C_{1}\left(A C_{2}\right)^{\kappa}\right| \phi_{b}^{n}(b-r)-\left.b\right|^{\kappa} .\right.
$$

Since $\phi_{b^{n}}\left(B_{-}(b, r)\right)=B_{-}\left(b, b-\phi_{b}^{n}(b-r) \mid\right)$ and since $\tau_{q} \neq b$, it follows from the Bounded Distortion Property and (6.3) that

$$
\begin{aligned}
m\left(\phi_{\tau b^{n}}\left(B_{-}(b, r)\right)\right) & =m\left(\phi_{\tau}\left(B_{-}\left(b, b-\phi_{b}^{n}(b-r)\right)\right)\right) \geq K^{-h}|| \phi_{\tau}^{\prime} \|^{h} m\left(B_{-}\left(b, b-\phi_{b}^{n}(b-r)\right)\right) \\
& \geq K^{-2 h}\left\|\phi_{\left.\tau\right|_{q-1}}^{\prime}\right\|^{h}\left\|\phi_{\tau_{q}}\right\|^{h} C_{1}^{-1}\left|\phi_{b}^{n}(b-r)-b\right|^{\kappa} .
\end{aligned}
$$

Combining this with (6.6) we get that

$$
m\left(\phi_{\tau b^{n}}\left(B_{+}(b, r)\right)\right) \leq A A_{1} C_{1}^{2}\left(A C_{2}\right)^{\kappa} K^{2 h} m\left(\phi_{\tau b^{n}}\left(B_{-}(b, r)\right)\right)
$$

where $A_{1}=\max \left\{\left\|\phi_{i}^{\prime}\right\|: i \in I\right\} \min ^{-1}\left\{\inf \left\{\left|\phi_{i}^{\prime}(x)\right|: x \in X\right\}: i \in I\right\}$. So, we are done in the case when $\hat{J}$ is a proper subset of $X$.

So, assume that either (be) or (ce) holds. Consider again without loss of generality the righthand endpoint $b$ and then a word $\omega=\tau b^{n}, n \geq 0$. Put also $q=|\tau|$. If $\phi_{\tau_{q}}(b)$ is no endpoint of any segment $\phi_{i}(X), i \neq \tau_{q}$, then the same argument as in the previous case (ae) completes the proof. If $\phi_{\tau_{q}}(b)=\phi_{j}(b)$ for some $j \in I \backslash\left\{\tau_{q}\right\}$, then also exactly the same reasoning as in the case (ae) completes the proof. So, suppose that $\phi_{\tau_{q}}(b)=\phi_{j}(a)$ for some $j \in I$. Since, by the Open Set Condition, the sets $\phi_{\tau_{q}}(B(b, \operatorname{dist}(X, \partial V)))$ and $\phi_{j}(X)$ overlap with the common enddpoint $\phi_{\tau_{q}}(b)=\phi_{j}(a)$, there thus exists a unique positive-valued function $H_{a}$ defined on $[0, R]$ with $R>0$ sufficiently small, such that $\phi_{\tau_{q}}(b+s)=\phi_{j}\left(a+H_{a}(s)\right)$. Formula (6.5) remains true with $H_{b}$ replaced by $H_{a}$ if in the minimum part of the formula defining the constant $A, \phi_{i}^{\prime}(b)$ is replaced by $\phi_{i}^{\prime}(a)$. Because of (be) or (ce), which mean that both fixed points $a$ and $b$ are of the same kind, formula (6.3) remains the same with $b$ replaced by $a$, and (6.4) takes on the form

$$
C_{2}^{-1} \leq \frac{\left|\phi_{b}^{n}(b-s)-b\right|}{\left|\phi_{b}^{n}(a+s)-a\right|} \leq C_{2} .
$$

Similarly as in the case (ae), we therefore get that

$\phi_{\tau b^{n}}\left(B_{+}(b, r)\right) \subset \phi_{\left.\tau\right|_{q-1}} \circ \phi_{j}\left(B_{+}\left(a, A\left|\phi_{a}^{n}(a+r)-a\right|\right)\right) \subset \phi_{\left.\tau\right|_{q-1}} \circ \phi_{j}\left(B_{+}\left(a, A C_{2}\left|\phi_{b}^{n}(b+r)-b\right|\right)\right)$, and from this

$$
m\left(\phi_{\tau b^{n}}\left(B_{+}(b, r)\right)\right) \leq \| \phi_{\left.\tau\right|_{q-1}}^{\prime}||^{h}|| \phi_{j}^{\prime}||^{h} C_{1}\left(A C_{2}\right)^{\kappa}\left|\phi_{b}^{n}(b-r)-b\right|^{\kappa} .
$$

Since (6.7) is always true, independently of any case considered, combining it with (6.9), we obtain (6.8) also in this case ((be) or (ce)). We are done.

Combining Proposition 4.1, Lemma 6.1 Lemma 6.3, and Lemma 6.2, we obtain the following main result of this section. 
Theorem 6.4. If $S$ is a 1-dimensional finite extendable parabolic iterated function system, then the corresponding h-conformal measure satisfies the doubling property.

\section{Badly Approximable Points}

We start with the following.

Lemma 7.1. If $\mu$ is a Borel probability measure on the real line $\mathbb{R}$, satisfying the (efd) and the doubling property, then the measure $\mu$ is absolutely decaying.

Proof. In view of the doubling property and the (efd) property, there are constants $C>0$ and $\alpha \in(0,1)$ such that for all $r>0$ sufficiently small and all $z \in \operatorname{supp}(\mu)$, we have $\mu(B(z, 2 r)) \leq C \mu(B(z, r))$ and $\mu(B(z, r)) \leq \alpha \mu(B(z, 2 r))$. In order to prove the lemma, fix $x \in \operatorname{supp}(\mu), y \in B(x, r)$ and $\varepsilon \in(0,1 / 4)$. If $B(y, \varepsilon r) \cap \operatorname{supp}(\mu)=\emptyset$, then $\mu(B(y, \varepsilon r))=0$ and we are done. Otherwise, take an arbitrary point $w \in B(y, \varepsilon r) \cap \operatorname{supp}(\mu)$. Then $B(y, \varepsilon r) \subset$ $B(w, 2 \varepsilon r)$ and $B(w, r / 2) \subset B(x, 2 r)$. Let $n$ be the least integer such that $2^{n} \leq(4 \varepsilon)^{-1}$. Since $\varepsilon \in(0,1 / 4)$, we see that $n \geq 0$. Then $(2 \varepsilon r) 2^{n} \leq r / 2$, and consequently

$$
\mu(B(y, \varepsilon r)) \leq \mu(B(w, 2 \varepsilon r)) \leq \alpha^{n} \mu(B(w, r / 2)) \leq \alpha^{n} \mu(B(x, 2 r)) \leq C \alpha^{n} \mu(B(x, r)) .
$$

Now, by the definition of $n$, we have $2^{n} \geq(8 \varepsilon)^{-1}$. Hence, as $\log \alpha<0$,

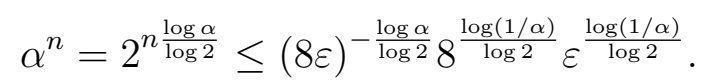

Therefore

$$
\mu(B(x, r) \cap B(y, \varepsilon r)) \leq \mu(B(y, \varepsilon r)) \leq C 8^{\frac{\log (1 / \alpha)}{\log 2}} \varepsilon^{\frac{\log (1 / \alpha)}{\log 2}} \mu(B(x, r)),
$$

and we are done.

Set

$$
p_{S}=\max \left\{p_{i}: i \in \Omega\right\}
$$

Proposition 7.2. If $S$ is a finite 1-dimensional parabolic iterated function system satisfying condition (a) of Proposition 4.1, then the corresponding h-conformal measure $m$ is $h+p_{S}(h-$ 1)-upper geometric.

Proof. We infer from condition (a) of Proposition 4.1 that for all $r>0$ small enough, all $x \in X\left((X, V)\right.$ is the $S$-eligible pair involved in condition (a)), and all $\omega \in I^{*}$,

$$
m\left(\phi_{\omega}(B(x, r))\right) \leq(C+1) m\left(\phi_{\omega}(B(x, r)) \cap X\right) .
$$

This is the property that makes the proofs of Lemma 6.3 and Theorem 6.4, which is the same as Theorem 6.2 in [7], from [6] go through in the setting of our proposition. As the result, we get that there exists a constant $Q \geq 1$ such that for every $r>0$ sufficiently small and every 
$z=\pi(\omega) \in J_{S}$ there exist a number $p(z, r) \in\left\{p_{i}: i \in \Omega\right\} \cup\{0\}$ and an integer $u \geq 0$ such that

$$
Q^{-1}\left(r^{-1}\left|\phi_{\left.\omega\right|_{u}}^{\prime}\left(\pi\left(\sigma^{u}(\omega)\right)\right)\right|\right)^{p(z, r)(1-h)} \leq \frac{m(B(z, r))}{r^{h}} \leq Q\left(r^{-1}\left|\phi_{\left.\omega\right|_{u}}^{\prime}\left(\pi\left(\sigma^{u}(\omega)\right)\right)\right|\right)^{p(z, r)(1-h)} .
$$

Since $\left|\phi_{\left.\omega\right|_{u}}^{\prime}\left(\pi\left(\sigma^{u}(\omega)\right)\right)\right| \leq 1$ and since $1-h \geq 0$, we therefore get from (7.1) that $m(B(z, r)) \leq$ $Q r^{h+p(z, r)(h-1)} \leq Q r^{h+p_{S}(h-1)}$ for all $r>0$ sufficiently small and every $z \in J_{S}$. The proof is complete.

As an immediate consequence of this, Proposition 7.2, Lemma 6.3, and Theorem 1.1 from [1], we get the following main result of this section.

Theorem 7.3. If $S$ is a finite extendable 1-dimensional parabolic iterated function system, then the Hausdorff dimension of badly approximable points in $J_{\Phi}$ is greater than or equal to $h+p_{S}(h-1)>0$.

Note that because of Theorem $2.1, h+p_{S}(h-1)>0$

\section{REFERENCES}

[1] D. Kleinbock, B. Weiss, Badly approximable vectors on fractals, Selecta Math. 10 (2004), 479-523.

[2] D. Kleinbock, E. Lindenstrauss, B. Weiss, On fractal measures and Diophantine approximation, Preprint 2003

[3] R. D. Mauldin, M. Urbański, Parabolic iterated function systems, Ergod. Th. \& Dynam. Sys. 20 (2000), 1423-1447.

[4] D. Mauldin, M. Urbański, Fractal measures for parabolic IFS, Adv. in Math. 168 (2002), 225-253.

[5] D. Mauldin, M. Urbański, The Doubling Property of Conformal Measures of Infinite Iterated Function Systems, J. Numb. Theory, 102 (2003), 23-40.

[6] M. Urbański, Parabolic Cantor sets, Preprint 199?, available on Urbański's webpage.

[7] M. Urbański, Parabolic Cantor sets, Fund. Math. 151 (1996), 241-277.

[8] M. Urbański, Diophantine Approximation for Conformal Measures of One-Dimensional Iterated Function Systems, Preprint 2003, to appear Compositio Math.

[9] M. Urbański, Diophantine Approximation of Self-Conformal Measures, Journal Number Th. 110 (2005) 219-235

[10] B. Weiss, Almost no points on a Cantor set are very well approximable, R. Soc. Lond. Proc. Ser. A Math. Phys. Eng. Sci. 457 (2001), 949-952.

Mariusz Urbański; Department of Mathematics, University of North Texas, P.O. Box 311430, Denton TX 76203-1430, USA

urbanski@unt.edu, http://www.math.unt.edu/ urbanski 